\title{
IMPACTOS NO COMPORTAMENTO DO FRETE: UMA APLICAÇÃO DE EQUILÍBRIO GERAL COMPUTÁVEL PARA OS PRODUTOS AGROPECUÁRIOS DO BRASIL
}

\author{
${ }^{1}$ Guilherme Asai, Carlos Alberto Piacenti, ${ }^{\Omega}$ Angelo Costa Gurgel \\ Universidade Estadual do Oeste do Paraná - UNIOESTE, Cascavel, PR (Brasil) \\ $\Omega$ Fundação Getúlio Vargas - FGV, São Paulo, SP (Brasil)
}

\section{DETALHES DO ARTIGO}

\section{Histórico do Artigo:}

Recebido: 08 de Julho de 2019

Aceito: 01 de Junho de 2020

Disponível online: 01 de Agosto de 2020

Sistema de revisão "Double blind review"

Editor Científico

Ilan Avrichir

\section{Palavras-chaves:}

Equilíbrio Geral Computável

Logística

Frete

Agropecuária

\begin{abstract}
RESUMO
Objetivo: este trabalho teve como objetivo investigar o impacto da oscilação do custo do frete para os produtos agropecuários brasileiros.

Método: trata-se de uma pesquisa descritiva de natureza quantitativa, focado na análise de cenário por meio da modelagem em equilíbrio geral computável para as cinco macrorregiões brasileiras.

Principais resultados: como resultado da pesquisa indica-se: (i) a importação e a exportação sofreram impacto causado pela variação do preço do frete, aumentando em todas as macrorregiões brasileiras; (ii) o aumento das importações e exportações é um indicativo no movimento dos fluxos comerciais e traz benefícios para a economia do país, favorecendo a troca e a economia regional e (iii) as regiões com menor custo de frete, apresentam ganhos de competitividade para produtos agropecuários.

Relevância/originalidade: trabalho contribui com uma agenda de pesquisa que envolve setores importantes da economia brasileira - agropecuária e transportes e como estão relacionados entre si, fornecendo uma visão de como o custo de frete influência no comércio dos produtos agropecuários no Brasil.

Contribuições teóricas/metodológicas: o trabalho apresenta uma abordagem diferente de métodos mais tradicionais, por ECG, criando uma alternativa para análise de como o custo de transporte impacta no comércio inter-regional.
\end{abstract}

\section{Introdução}

No final dos anos 1990 e início dos anos 2000, o mundo globalizado e a era da internet acarretou comodidades e facilidades para as pessoas. $\mathrm{O}$ acesso a informações, mobilidade, produtos e outros bens é facilitado pelo comércio globalizado e o ato de compra foi simplificado. Todos os produtos físicos devem ser entregues ao comprador em determinado tempo e com segurança, se o produto for virtual, como um software, por exemplo, a entrega pode ser através da rede de computadores, mas nem todo produto é assim, a maioria requer uma entrega física.

A entrega de produtos físicos é atribuição da logística que Antón (2005) trata como uma ciência em que se estuda como as mercadorias excedem o tempo e a distância de forma eficiente para manter uma atividade produtiva. Assim, Rutner e Langley (2000), Bosona e Gebresenbet (2013) e Rushton et al.
(2014) entenderam a logística como o elo entre produtores e consumidores, ou vice-versa, com garantia do fluxo de produtos e serviços do ponto de origem ao ponto de consumo.

De certa forma, a logística pode ser confundida ou usada como sinônimo de transporte. Crainic e Laporte (1996) observam o transporte como uma atividade que apoia as demais atividades econômicas e sociais e viabiliza a troca. Por sua vez, Cranic (2003) coloca o transporte como um componente-chave da cadeia de suprimentos, que inclui um movimento de matérias-primas para a produção até os produtos acabados.

A logística, em geral, é responsável por integrar negócios segundo a definição de Cooper et al. (1997). De acordo com isso, Christopher (2016) destaca que a logística planeja operações e as estrutura seguindo informações capazes de gerar ganhos de eficiência 
para o negócio e assim, obter vantagens competitivas.

Uma forma de gerar eficiência e competitividade é no transporte, ou seja, no custo do frete. Negócios cujo frete tenha um valor diferenciado, pode se tornar um atrativo, tornando-o mais competitivo que os demais. De fato, a redução do frete pode garantir um baixo custo de produção ou até mesmo um menor custo de entrega das mercadorias o que pode viabilizar um menor preço do bem em questão. Em um mundo de estreita margem de lucro, como as commodities agrícolas, reduzir os custos pode implicar na sobrevivência de alguma empresa no mercado.

Para determinar a taxa de frete, algumas transportadoras se baseiam em fatores como velocidade de entrega, tipo de produto e distância. Todas essas variáveis são importantes quando o produto é perecível, como produtos agropecuários ou alimentícios. Piercy e Ballou (1978) estudam atributos que impactam no valor do frete e tiveram como variáveis mais significativas: a velocidade e o tempo (tempo de trânsito), além da disponibilidade de serviço. Logo, os autores indicam que a melhor forma de se efetuar o transporte de carga é aliar essas variáveis para fornecer uma alternativa de transporte com menor custo de frete.

No caso do Brasil, o meio de transporte em que se alia essas duas características é o rodoviário, em especial se tratando de produtos agropecuários (BRANCO et al. 2012). Dessa forma, Nascimento et al. (2010) e Perá et al. (2013) indicam o frete como o principal custo de transporte de cargas que é diretamente influenciado, entre outros fatores, pela infraestrutura das vias de transporte. Indo além, Perá et al. (2013) representa que o combustível é um fator de impacto quando a qualidade da via implica na redução do tempo de viagem e na diminuição do consumo de combustível, contribuindo com o menor custo de frete.

O frete é uma variável que depende do local, do produto, da periodicidade, da perecibilidade eo meio de transporte utilizado que impacta diretamente no custo final do produto e deve ser considerado na decisão de comprar ou não determinado produto de um determinado produtor e local. Isto influencia diretamente na competitividade de um produto, ou seja, um frete competitivo representando a entrada de negócios em mercados disputados.
A demanda pelo transporte ocorre porque existe diferença nas localidades geográficas entre produtores (vendedores) e consumidores (compradores). No entanto, o cálculo do frete não é uma ciência exata e é difícil prever seu comportamento a longo prazo. Por isso, torna-se importante estudar o comportamento do frete e os impactos que sua volatilidade ocasiona nos comércios, principalmente os mais disputados e como margem de lucro pequena.

Em linha com o comportamento do frete, países com atividade econômica de importação e exportação elevada, como o Brasil, podem ser afetados pelo aumento e diminuição do frete internacional. A oscilação no valor do frete pode impactar na competitividade do país em alguns setores econômicos, tornando o produto caro demais para outros mercados.

Assim, este artigo pretende entender o que acontece com o consumo de mercadorias (preço, exportação e importação) para produtos agropecuários devido à variação do frete. Logo, como objetivo principal deste trabalho propõe-se um estudo para o Brasil utilizando um Modelo de Equilíbrio Geral Computável (EGC) que investigará o impacto da oscilação do custo do frete para os produtos agropecuários brasileiros.

Para investigar o impacto do comportamento do frete nos produtos agropecuários brasileiros, foi escolhido o Projeto de Análise de Equilíbrio Geral da Economia Brasileira (PAEG) que retrata a economia do Brasil em 19 setores (7 relacionados a produtos agrícolas e consumo de alimentos) e 12 regiões (cinco macrorregiões do Brasil e outras sete regiões). A proxy de custo do frete se dará com base na oscilação do custo do combustível, uma vez existe a predominância do transporte de cargas pelo modal rodoviário.

O trabalho está estruturado em cinco capítulos, incluindo este de introdução. O segundo capítulo trata um breve panorama do transporte de cargas no Brasil. A terceira parte é dedicada à metodologia utilizada nesse trabalho. A quarta parte se dedica a apresentar os resultados e os impactos causados pelo custo de transporte. Por fim, o quinto capítulo é dedicado às considerações finais. 


\section{Transporte de cargas agropecuárias no Brasil}

As necessidades da logística em encontrar meios de ligar os agentes de mercado recaem sobre os atores - produtores, expedidores, carries, governos e consumidores - que dependem dos serviços de transporte para ligá-los entre si. A ligação entre os atores mencionados faz com que o serviço de tenha papel fundamental numa cadeia produtiva (DASKIN, 1985).
No Brasil, existe a predominância e relativa dependência do transporte de cargas através das estradas (modal rodoviário) muito pela falta de infraestrutura e disponibilidade dos demais modais de transportes. Porém a existência e a utilização dos modais ferroviário e hidroviário podem contribuir com a diminuição nos custos de transporte e aumentar a eficiência da logística no (CASTILLO, 2004; LIMA, 2006; KATO, 2007; KAWANO et al., 2012; BOZOKY et al., 2014; BRANCO et al., 2012).

A matriz de transporte brasileira está dividida como ilustrado na Figura 1.

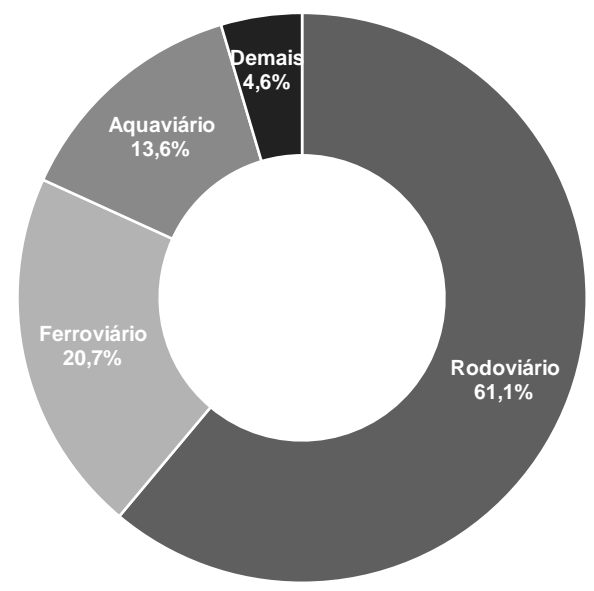

Figura 1. Matriz de transporte brasileira para o ano de 2014. Fonte: elaboração própria.

Nota: dados da Confederação Nacional do Transporte (CNT, 2014).

Vertendo-se o transporte de produtos agropecuários, Caixeta Filho (2010) observa que a organização espacial da produção torna o transporte fator importante na competitividade dos produtos justamente pela composição dos custos de produção, cuja espacialização das zonas produtoras, matérias primas e zona consumidora (até para exportação) aumentam os custos dos produtos agrícolas e agropecuários.

A importância dos transportes de produtos agropecuários torna-se mais crítica ao considerar a sensibilidade ao tempo dos mesmos.

Segundo Behar e Venables (2011), Attavanich et al. (2013) e Redding e Turner (2014) a perecibilidade dos produtos de origem vegetal e animal aumentam os custos de transporte, necessitando um maior cuidado e qualidade no serviço de transporte contratado, tornando este tipo de serviço mais restritivo perante que o transporte de outros produtos.
Do mesmo modo, a espacialidade da produção apontada por Wilson e Dahl (2011) que confere ao transporte de grãos a uma maior volatilidade de transporte na safra e na entressafra, tornando menos previsível o preço do frete e aumentando os custos de comercialização. Por causa disso a demanda por investimentos em infraestrutura adequada fica prejudicada.

Colaborando com a visão de Caixeta Filho (2010), Castro (2009) já comentava em seu estudo que o custo logístico é um componente relevante na composição do preço final dos produtos aos consumidores e com o aumento da malha rodoviária a partir de 1950, houve a contribuição para a expansão geográfica da produção agropecuária brasileira.

Considerando o modal rodoviário como principal meio de transporte de cargas no Brasil, o custo se deve diretamente aos elementos ligados na condução e capacidade de carga do veículo. Segundo 
Lewczuk e Wasiak (2011) os custos de transporte rodoviário derivam da distância (quilometragem percorrida), peso do produto e tempo de carregamento e descarregamento.

Oliveira et al. (2013) e Oliveira et al. (2016) apontam que o frete é diretamente influenciado pela relação do preço do combustível. No modal rodoviário, os autores reconhecem que o preço do diesel influencia na questão do custo logístico para o transporte de cargas, impactando no preço final do frete. A dependência do transporte de cargas agrícolas e agropecuárias pelo modal rodoviário e o aumento dos custos, provenientes deste tipo de transporte, acaba se tornando motivo de preocupação e alvo de estudo ao longo dos anos. Estudos como os de Fujita et al. (2000), Gurgel (2014) e Oliveira et al. (2015) relatam o custo de transporte como preocupação na logística e distribuição de produtos agrícolas e agropecuários.

Para entrega de produtos e mercadorias, o setor logístico brasileiro privilegia rodovias, porém outros meios de transporte estão recebendo novos investimentos e crescendo em participação (MARCHETTI e FERREIRA, 2012; ASAl et al., 2017; MACHADO et al., 2018). No entanto, para simular cenários de mudanças nos preços dos fretes este trabalho utilizará a flutuação dos preços dos combustíveis (diesel) como proxy, o que pode ser justificado devido ao elevado uso do modal rodoviário para transporte de cargas agropecuárias.

Com o panorama traçado do serviço de transporte de cargas agrícolas e agropecuárias no Brasil é possível explorar seus fatores limitantes que acarretam a perda de eficiência e da vantagem comparativa.

\section{Metodologia}

O modelo de Equilíbrio Geral Computável tem a capacidade de representar a economia de um país e suas relações comerciais com demais dentro de um conjunto de equações algébricas. Para Sadoulet e De Janvry (1995) e Partridge e Rickman (1998), os modelos EGC podem capturar as relações entre os agentes econômicos através dos aspectos macroeconômicos e microeconômicos presentes na matriz insumo-produto de cada país.

Dentre os diversos usos do ECG, a logística foi objeto de estudo de Tavasszy et al. (2003), Haddad (2004), Bröcker (2004), Barry (2013), Gurgel (2014),
Kim et al. (2017) e Chen (2018). Tais trabalhos avaliaram os impactos da logística de transportes na economia ora por investimentos no setor, ora por diminuição de custos. Os estudos apontam a estruturação de diversos modelos de EGC para avaliar a eficiência do setor ou avaliar os investimentos feitos na melhora da qualidade logística.

Haddad (2004) analisa os retornos obtidos ao se diminuir os custos de transportes e quanto estes fatores interferem no desenvolvimento regional. Neste estudo, existe a elaboração de um modelo próprio de EGC que permitiu verificar os ganhos de eficiência do transporte para um determinado estado do Brasil.

Outros trabalhos como os de Mendes (2013), Fagundes et al. (2015), Waquil (2019) avaliam que o impacto o comércio de produtos agropecuários é afetado pelo custo de transporte. Análogo aos trabalhos com EGC, estes autores utilizaram metodologias diferentes como análise espacial e matriz insumo-produto.

Logo, este trabalho contribui em fomentar o tema e será baseado em uma pesquisa descritiva de natureza quantitativa, focado na análise de cenários por meio da modelagem EGC, usada para investigar os impactos do comportamento do frete no comércio de produtos agropecuários brasileiros através das relações de trocas inter-regionais via importação e exportação dos produtos agropecuários das macrorregiões brasileiras em si e as demais regiões presentes no modelo. Adicionalmente, o impacto no fator preço dos produtos agropecuários será analisado.

3.1. Projeto de Análise de Equilíbrio Geral da Economia Brasileira

O PAEG é um modelo estático, multirregional e multisetorial, estruturado para representar a economia brasileira e suas interações com outras economias em todo o mundo. De acordo com Gurgel et al. (2011) cada região do modelo é representada por uma estrutura de demanda final onde produtor e consumidor atuam para maximizar seu bem-estar sujeito à restrição orçamentária, considerando investimento fixo, fluxo de capital e produção do setor público.

A estrutura do PAEG é baseada no GTAPInGAMS (Rutherford e Paltsev, 2000; Rutherford, 2005), adotando um problema de complementaridade não 
linear em General Algebraic Modeling System (GAMS) desenvolvido por Brooke et al. (1998). Para a linguagem de programação, o PAEG utiliza a sintaxe baseada em Modeling Programming system for General Equilibrium (MPSGE) estruturado por Rutherford (1999). O PAEG é dividido em um total de 12 regiões e 19 setores compatível com GTAPinGAMS na sétima versão, que se referem à economia mundial para o ano de 2011. Com o propósito de retratar a economia brasileira em suas cinco macrorregiões, o banco de dados do Global Trade Analysis Project (GTAP) foi desagregado conforme descrito por Teixeira et al. (2013). As regiões, setores e os fatores de produção considerados no PAEG estão descritos na Figura 2.

\begin{tabular}{|c|c|c|c|}
\hline Sectores & Acrônimo & Regiões & Acrônimo \\
\hline Arroz & $(p d r)$ & Norte do Brasil & Nor \\
\hline Milho e cereais & (gro) & Nordeste do Brasil & Nde \\
\hline Soja e outros óleos & (osd) & Centro-Oeste do Brasil & Coe \\
\hline $\begin{array}{l}\text { Cana-de-açúcar, beterraba e indústria } \\
\text { açucarada }\end{array}$ & (c_b) & Sudeste do Brasil & Sde \\
\hline Carne e gado & (oap) & Sul do Brasil & SUL \\
\hline Leite e laticínios & $(\mathrm{rmk})$ & Resto do Mercosul & Rms \\
\hline Produtos do agronegócio & (agr) & Venezuela & VEM \\
\hline Alimentos & $($ foo $)$ & Estados Unidos & Eua \\
\hline Indústria Têxtil & (tex) & Resto do Nafta & RNF \\
\hline Roupas e sapatos & (wap) & Resto da América & Roa \\
\hline Madeira e móveis & (lum) & Europa & Euros \\
\hline Celulose e indústria grafica & (ppp) & China & Chn \\
\hline $\begin{array}{l}\text { Indústria química, plástica e de borracha } \\
\text { Fabricados }\end{array}$ & $\begin{array}{l}\text { (crp) } \\
\text { (homem) }\end{array}$ & Resto do mundo & Linha \\
\hline Distribuição de gás, eletricidade e água & (siu) & Fatores de Produção & Acrônimo \\
\hline Edifício & (cns) & Capital & Tampa \\
\hline Vendas & $(\operatorname{trd})$ & Trabalho & Laboratório \\
\hline Transporte & (otp) & & \\
\hline Serviço e administração pública & (adm) & & \\
\hline
\end{tabular}

Figura 2. Regiões e setores presentes no modelo PAEG. Fonte: Pinto, et al., 2016.

Gurgel et al. (2011) e Teixeira et al. (2013) definem o modelo PAEG como uma forma de representar os bens e serviços produzidos nas economias brasileira e mundial. As regiões são representadas por uma estrutura de demanda final e o comportamento dos agentes é um otimizador na forma de maximizar o bem-estar. Esses autores indicaram que os setores produtivos minimizam seus custos com uma combinação de insumos intermediários e fatores primários, dada o uso de uma certa tecnologia. Ainda fazem parte do modelo o comércio bilateral, custos de transporte, impostos e subsídios. Um conjunto de banco de dados do PAEG é indicado na Figura 3.

\begin{tabular}{|ll|}
\hline Índice & Descrição \\
\hline $\mathrm{I}, \mathrm{j}$ & Setores e bens \\
\hline $\mathrm{r}, \mathrm{s}$ & Países e regiões \\
\hline $\mathrm{f} \in \mathrm{m}$ & $\begin{array}{l}\text { Fatores de Produção Livre da Mobilidade entre uma determinada região: mão-de- } \\
\text { obra; Capital }\end{array}$ \\
\hline $\mathrm{f} \in \mathrm{s}$ & Fatores de Produção Fixa: Recursos naturais \\
\hline
\end{tabular}

Figura 3. Conjunto de dados presentes no PAEG.

Fonte: Gurgel et al. 2011.

Para estruturar o modelo em economias regionais, algumas variáveis são criadas, partindo de um modelo econômico. As variáveis do modelo são: (i) $Y_{i r}$,como produção do bem i na região $r$; (ii) $C_{r}$, $I_{r}$ e
$\mathrm{G}_{\mathrm{r}}$ são o consumo privado, investimento e consumo público na região $r$, respectivamente; (iii) $M_{j r}$ representa a importação de bem j pela região $r$; (iv) o $\mathrm{HH}_{\mathrm{r}}$ é a variável para consumidor representativo; (v) 
setor público ou governo na região $r$ é a variável $\mathrm{GOVT}_{r}$; e (vi) $\mathrm{FT}_{\mathrm{sr}}$ representa a atividade através da qual os insumos específicos são alocados para setores regional está estruturada no modelo PAEG a Figura 4 ilustra a estrutura e os fluxos econômicos.

privados. Para exemplificar como a economia

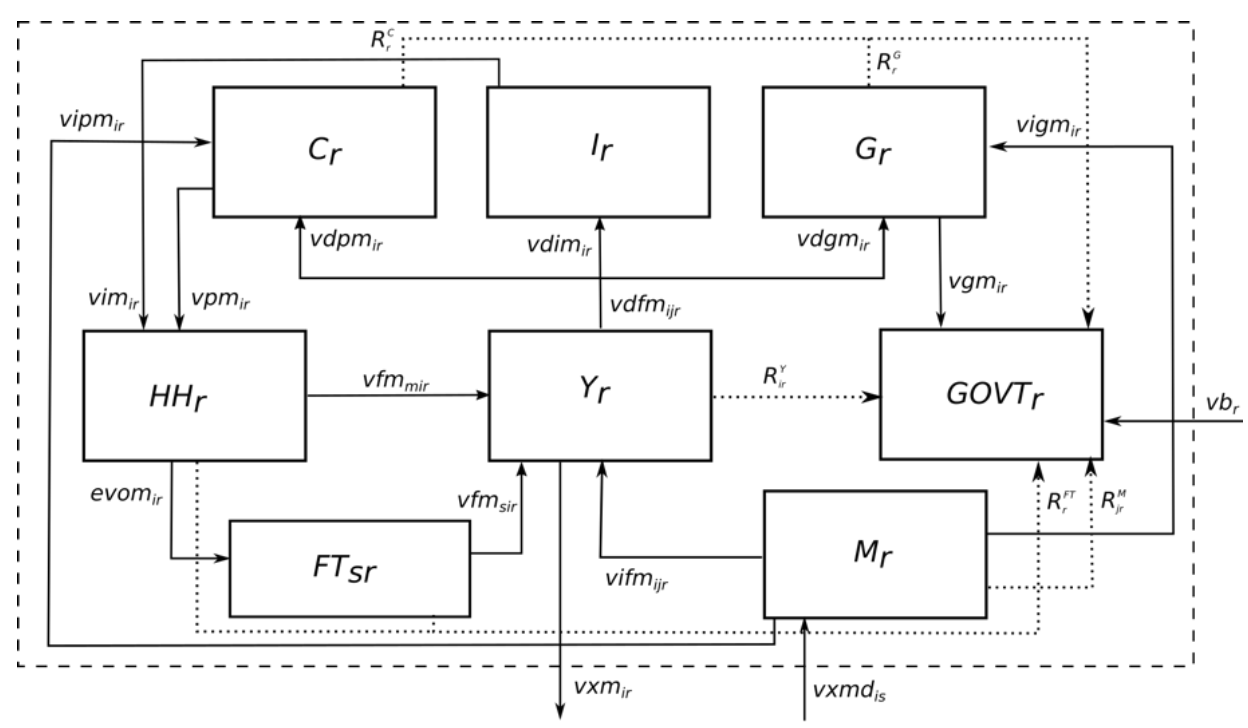

Figura 4. Estrutura econômica regional e fluxo econômico do modelo PAEG.

Fonte: Pinto, et al., 2016.

Na Figura 4, a linha tracejada define uma região $r$, a linha sólida indica os fluxos das variáveis e as linhas pontilhadas determinam o fluxo fiscal. Além disso, é possível ver as variáveis - $\mathrm{Y}_{\mathrm{i}}, \mathrm{C}_{\mathrm{r}}, \mathrm{I}_{\mathrm{r}}, \mathrm{G}_{\mathrm{r}}, \mathrm{M}_{\mathrm{jr}}$ e $\mathrm{FT}_{\mathrm{sr}}$ - - e parâmetros. Esses outros parâmetros são os impostos indiretos que representam para a letra "R" e afeta: produção $\left(R_{i r}^{Y}\right)$; consumo $\left(R_{r}^{C}\right)$; demanda pública $\left(R_{r}^{G}\right)$; fatores de produção $\left(R_{r}^{F T}\right)$; e importações $\left(R_{j r}^{M}\right)$.

Além disso, a Figura 4 traz a identidade contábil do PAEG para os agentes e como cada um afeta o fluxo comercial. Teixeira et al. (2013) define o modelo PAEG como uma concorrência perfeita e retornos constantes à escala, com custos intermediários de insumos e fatores de produção iguais ao valor de produção e, assim, lucros econômicos iguais a zero. Para cumprir todas essas condições, a identidade contábil das variáveis do modelo é indicada nas Equações 1 a 7.

$$
\begin{gathered}
Y_{\text {ir: }}: \quad \sum_{f} v f m_{f i r}+\sum_{j}\left(v i f m_{j i r}+v d f m_{j i r}\right)+ \\
R_{i r}^{Y}=v o m_{i r} \\
\text { (Equação 1) } \\
\mathrm{M}_{\mathrm{ir}:}: \quad \sum_{s}\left(v x m d_{i s r}+\sum_{j} v t w r_{j i s r}\right)+R_{i r}^{M}=v i m_{i r} \\
\text { (Equação 2) }
\end{gathered}
$$$$
\mathrm{C}_{\mathrm{r}}: \quad \sum_{i}\left(v d p m_{i r}+v i p m_{i r}\right)+R_{i r}^{C}=v p m_{r}
$$$$
\text { (Equação 3) }
$$

$$
\begin{aligned}
& \mathrm{G}_{\mathrm{r}}: \quad \sum_{i}\left(v d g m_{i r}+v i g m_{i r}\right)+R_{i r}^{G}=v g m_{r} \\
& \text { (Equação 4) } \\
& \text { Ir } \quad \sum_{i} v \operatorname{dim}_{i r} \quad=\operatorname{vim}_{r} \quad(\text { Equação 5) } \\
& \mathrm{FT}_{\mathrm{fr}}: \quad \text { evom }_{f r}=\sum_{i} v f m_{f i r} \quad f \in S \\
& \text { (Equação 6) }
\end{aligned}
$$

$\mathrm{YT}_{\mathrm{j}}: \quad \sum_{r} v s t_{j r}=v t_{j}=\sum_{i r s} v t w r_{j i r s}$

(Equação 7)

Apresentada as identidades contábeis das variáveis presentes no modelo, Gurgel et al. (2011) descreve como essas identidades contábeis são calculadas e como são compostas, sendo: produção fatores de produção móveis (vifmijr, $f \in \mathrm{m}$ ); doméstica $\left(v o m_{i r}\right)$; exportação $\left(v x m d_{i r s}\right)$; serviços internacionais de transporte $\left(v s t_{i r}\right)$; demanda intermediária $\left(v d f m_{i j r}\right)$; consumo privado $\left(v d p m_{\text {ir }}\right)$; consumo governamental $\left(v d g m_{\text {ir }}\right)$; investimento $\left(\right.$ vim $\left._{i r}\right)$; bens importados $\left(\right.$ vim $\left._{\text {ir }}\right)$; bens utilizados no intermediário consumo (vifm ijr $_{\text {r }}$; consumo privado $\left(\right.$ vipm $_{\text {ir }}$ ); consumo governamental $\left(v_{\text {igm }}\right.$ ir $)$; consumo de agente público $\left(\right.$ vigm $\left._{\text {ir }}\right)$; exportação do bem i pela região $r\left(v x m_{i r}\right)$; importações de bem i por região $r\left(v x m d_{i r s}\right)$; serviços internacionais de transporte $\left(v t_{j}\right)$; valor da exportação de serviços de transporte $\left(v s t_{j r}\right)$; fluxos 
bilaterais de serviços de transporte adquiridos na importação de mercadorias $\left(v t w r_{j i s r}\right)$; restrição orçamentária do governo $\left(v g m_{r}\right)$; e restrição orçamentária do agente representativo $\left(\mathrm{evom}_{\mathrm{fr}}\right)$.

Com o propósito de cumprir o objetivo proposto, de analisar o impacto da oscilação do custo do frete para os produtos agropecuários brasileiros, adaptouse o modelo PAEG para refletir o custo de transporte.

Para entender os cenários propostos foi necessário estruturar algumas das identidades contábeis a fim de que retratem o custo do frete. Sendo, $\mathrm{vt}_{\mathrm{j}}$, o custo de transporte de bens e serviços de uma região, explorou-se essa variável para projeção de cenário. Logo, identidade que relacionada o frete é a descrita pela 7 e possui duas partes principais representadas nas Equações 8 a 9.

$\mathrm{vt}_{\mathrm{j}}=\sum_{\mathrm{r}} \mathrm{vst}_{\mathrm{jr}}$

(Equação 8)

$\mathrm{vt}_{\mathrm{j}}=\sum_{\mathrm{r}} \mathrm{vtwr}_{\mathrm{jisr}}$

(Equação 9)

A Equação 8 representa o equilíbrio dos serviços de transporte, fornecidos pela igualdade da oferta agregada de serviços de transporte e pelo valor dos serviços de transporte. Enquanto a Equação 9 descreve uma igualdade de oferta e demanda pelos serviços de transporte.

Essas duas equações estão diretamente correlacionadas com a função de produção descrita na Equação 1. A relação entre os serviços de transporte e a função de produção é baseada na identidade contábil na Equação 1 que também pode ser descrita de acordo coma Equação 10.

$$
\begin{aligned}
& \operatorname{vom}_{i r}=\sum_{\mathrm{r}} \mathrm{vxmd}_{\mathrm{irs}}+\sum_{\mathrm{j}} \mathrm{vdfm}_{\mathrm{ijr}}+\mathrm{vst}_{\mathrm{ir}}+ \\
& \mathrm{vdpm}_{\mathrm{ir}}+\mathrm{vdgm}_{\mathrm{ir}}+\operatorname{vdim}_{\mathrm{ir}}
\end{aligned}
$$

10)

Através da Equação 10, a produção é composta pela exportação $\left(v x m d_{i r s}\right)$, demanda intermediária $\left(v d f_{i j r}\right)$, consumo privado $\left(v d p m_{i r}\right)$, consumo governamental $\left(v d g m_{i r}\right)$, investimentos $\left(v \operatorname{dim}_{i r}\right) \mathrm{e}$ também pelos serviços de transporte (vst $\left.t_{i r}\right)$. Portanto, os bens e serviços são diretamente afetados pelo custo de transporte no modelo.

A lógica do modelo PAEG e suas identidades contábeis estão relacionadas ao MPSGE como blocos de produção. A Figura 5 mostra a árvore de decisão tecnológica representando o bloco de produção e descreve as tecnologias empreendidas pelas empresas, $v_{\text {or }}$, no PAEG.

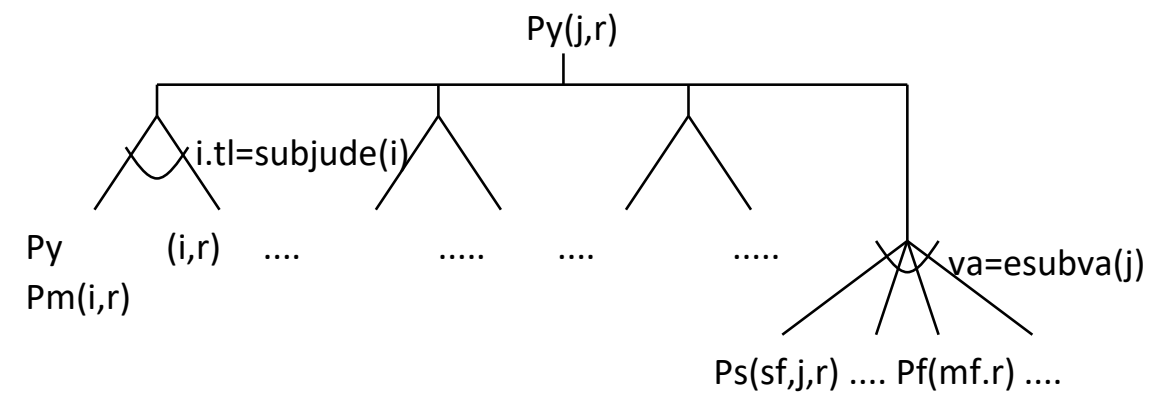

Figura 5. Árvore de decisão tecnológica da função de produção do modelo PAEG. Fonte: Gurgel et al., 2011.

Da mesma lógica para a função de produção, os serviços de transporte possuem uma árvore de decisão tecnológica representando a Figura 6.

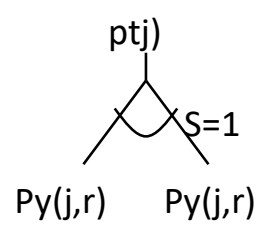

Figura 6. A árvore de decisão tecnológica da função de serviços de transporte modelo PAEG. Fonte: Gurgel et al., 2011. 
O bloco de produção para serviços de transporte é uma combinação dos insumos py(j,r) para produzir opt(j). Uma interpretação que pode ser dada a este bloco de produção é que para produzir pt(j) há necessidade de uma quantidade de insumos intermediários (i) que devem ser transportados (q) para obter o produto final (o). A lógica do modelo representa a matriz na Equação 11.

\$prod:yt(j)\$vtw(j) s: 1

o: $p t(j) \quad q: \operatorname{vtw}(j)$

i: $p y(j, r) \quad q: \operatorname{vst}(j, r)$

(Equação 11)

Neste caso, o preço dos serviços de transporte para determinadas regiões está em equilíbrio e nenhum excedente ou ausência são observados devido à elasticidade imposta pela função de Leontief, s:1. Para adaptar o PAEG a fim de que se capture as alterações do comportamento do frete, o vst será decomposto em duas outras variáveis: preço e quantidade de serviços de transporte. A Equação 12 introduz essas duas variáveis no modelo.

$S_{j}=D_{j r}=P_{i r} \times Q_{\text {ir }}$

(Equação 12)

Em equilíbrio.

$\mathrm{vst}_{\mathrm{ir}}=\mathrm{P}_{\mathrm{ir}} \times \mathrm{Q}_{\mathrm{r}}$

(Equação 13)

Assim, o preço de como insumo na região $\mathrm{P}_{\mathrm{ijr}} \mathrm{e}$ demanda da quantidade desse insumo em outra região $\left(Q_{i j r}\right)$ dos serviços de transporte é dado de acordo com a lei de oferta e demanda. Portanto, a volatilidade do frete será simulada em mudanças desse preço, o que cria cenários para investigar o impacto da taxa de frete nos fluxos comerciais brasileiros, restringindo aos produtos agropecuários.

Para este trabalho, aos setores agropecuários e alimentícios são: rice(pdr); milho e cereais (gro); soja e outros óleos (osd); cana-de-açúcar, beterraba e indústria açucarada (c_b); carne e pecuária (oap); milk e laticínios (rmk); outros produtos agrícolas (agr); e alimentos (foo).

Nesse caso, todo cenário projetado será baseado em alterações na taxa de frete, o que leva a diferentes quantidades exigidas para cada produto e setor ( $i$ e j) divisões por cada região presente no modelo e aumento no custo de transporte. A alteração se valerá da oscilação do preço do combustível para cada macrorregião, uma vez que o impacto do preço do diesel se demonstra relevante para o valor do frete. Assim, levou-se em consideração o valor médio dos combustíveis de 2013 a 2019.

Para projetar o cenário que traduzem o impacto do custo de transporte no comércio de produtos agropecuários brasileiros utilizou-se a variação do frete para realizar os choques no modelo de EGC e assim projetar os impactos do frete nas importações e exportações e o preço, cujas variáveis representarão o impacto no comércio inter-regional.

Dito isso, levantaram-se os preços do diesel para - uso em caminhões para cada macrorregião brasileira entre janeiro de 2013 e fevereiro de 2019, a fim de determinar uma magnitude para o choque no modelo EGC. Foram utilizados os preços médios para consumidores, ou seja, os preços finais com todos os impostos incluídos. Os resultados dos preços dos combustíveis estão presentes na Tabela 1.

\begin{tabular}{cccccc}
\hline Ano & Centro-oeste & Nordeste & Norte & Sudeste & Sul \\
\hline 2013 & 2.605 & 2.348 & 2.478 & 2.427 & 2.420 \\
\hline 2014 & 2.818 & 2.567 & 2.716 & 2.636 & 2.629 \\
\hline 2015 & 3.137 & 2.953 & 3.117 & 2.935 & 2.915 \\
\hline 2016 & 3.336 & 3.145 & 3.361 & 3.107 & 3.038 \\
\hline 2017 & 3.457 & 3.164 & 3.463 & 3.232 & 3.103 \\
\hline 2018 & 3.771 & 3.560 & 3.743 & 3.568 & 3.438 \\
\hline 2019 & 3.710 & 3.569 & 3.757 & 3.507 & 3.386 \\
\hline $\begin{array}{c}\text { Média da taxa de } \\
\text { crescimento (\%) }\end{array}$ & 6.155 & 7.373 & 7.284 & 5.863 & 6.614 \\
\hline
\end{tabular}

Tabela 1. Diesel S10 preços por região em R\$ por litro. 
Com o valor do choque no modelo EGC calculado, cada macrorregião terá seu próprio multiplicador na taxa de preços $\mathrm{P}_{\mathrm{ir}}$, descrito na Equação 13, representando uma oscilação dos preços do frete. Deste ponto, nota-se que o preço do combustível subiu em todas as regiões, aumentando o valor de vst $_{\text {ir. }}$.

Portanto, no choque para a projeção do cenário tem-se o aumento do preço dos serviços de transportes, cuja magnitude é diferente em cada macrorregião. O nível de choque que representa cada região é descrito nas Equações 14 a 19.

$$
\begin{array}{lll}
\text { COE: } & \text { vst }_{\text {ir }}=\left(1,0615 \times \mathrm{P}_{\mathrm{ir}}\right) \times \mathrm{Q}_{\mathrm{r}} & \text { (Equação 14) } \\
\text { NOR: } & \text { vst }_{\text {ir }}=\left(1,0724 \times \mathrm{P}_{\mathrm{ir}}\right) \times \mathrm{Q}_{\mathrm{r}} & (\text { Equação 15) } \\
\text { NDE: } & \text { vst }_{\text {ir }}=\left(1,0737 \times \mathrm{P}_{\mathrm{ir}}\right) \times \mathrm{Q}_{\mathrm{r}} & (\text { Equação 16) } \\
\text { SUL: } & \text { vst }_{\text {ir }}=\left(1,0661 \times \mathrm{P}_{\mathrm{ir}}\right) \times \mathrm{Q}_{\mathrm{r}} & (\text { Equação 17) } \\
\text { SDE: } & \text { vst }_{\text {ir }}=\left(1,0586 \times \mathrm{P}_{\mathrm{ir}}\right) \times \mathrm{Q}_{\mathrm{r}} & \text { (Equação 18) }
\end{array}
$$

Com o aumento do transporte e, tendo como único fator de impacto a oscilação no valor com combustível, espera-se analisar os impactos nas importações, exportações e preço dos produtos agropecuários produzidos nas macrorregiões brasileiras. Vale ressaltar que a mudança do preço do frete somente será incidida no caso de serviços de transportes provenientes do Brasil.

\section{Impactos do comportamento do frete no comércio de produtos agropecuários}

Para analisar o impacto do frete no comércio inter-regionais dos produtos agropecuários, este trabalho terá como foco as importações, exportações e preços. No modelo, as exportações e importações retratam as trocas entre as macrorregiões brasileiras de forma a representar o nível de atividade do comércio para cada um dos produtos agropecuários em determinada macrorregião. Já os preços indicam o valor pago pelos produtos em suas respectivas regiões. Todos os resultados dos projetados estão presentes nas Tabelas 2, 3 e 4 .

\begin{tabular}{lccccc}
\hline & Norte & Nordeste & Centro-oeste & Sudeste & Sul \\
\hline Pdr & -0.743 & 0.065 & 0.565 & 0.719 & -0.214 \\
\hline Gro & -0.122 & 0.070 & 0.134 & 0.560 & 0.058 \\
\hline Osd & -0.177 & 0.079 & 0.392 & 0.662 & -0.220 \\
\hline c_b & -0.526 & 0.008 & 0.212 & 0.506 & 0.015 \\
\hline Oap & -0.186 & 0.066 & 0.302 & 0.690 & -0.044 \\
\hline rmk & -0.790 & -0.633 & -0.014 & 0.979 & -0.324 \\
\hline Agr & 1.167 & 0.389 & 0.409 & 1.124 & 0.406 \\
\hline Foo & -0.175 & 0.121 & 1.057 & 1.103 & 2.490 \\
\hline
\end{tabular}

Tabela 2. Variação das importações totais (\%).

Para o cenário projetado, a partir das importações, todas as regiões apresentam comportamento semelhante, bem como nas exportações. No entanto, em alguns setores, as importações não estão próximas das exportações, o que pode representar um desequilíbrio na balança comercial da região. O maior aumento das importações frente as exportações indicam que a região necessita de um complemente em sua produção local para suprir o consumo, enquanto o aumento das exportações representa uma auto suficiência.
Nos setores agrícolas, representados pelas commodities de arroz, milho e cereais, soja, açúcar, leite e carnes, as importações, em alguns setores, como o leite e derivados, há o aumento maior que as exportações que revela um indício de necessidade de complementar o consumo local com vindas de produtos de outras regiões.

Ao ter as importações superiores as exportações, pode-se dizer que já perda de autossuficiência do setor na região. 


\begin{tabular}{lccccc}
\hline & Norte & Nordeste & Centro-oeste & Sudeste & Sul \\
\hline Pdr & 3.401 & 3.144 & 0.278 & 3.246 & 1.337 \\
\hline Gro & 1.060 & 0.780 & 0.633 & 0.549 & 0.683 \\
\hline Osd & 1.784 & 1.622 & 0.689 & 0.825 & 1.444 \\
\hline c_b & 1.547 & 0.234 & 0.424 & -0.427 & 0.485 \\
\hline Oap & 1.057 & 0.529 & 0.447 & 0.200 & 0.872 \\
\hline rmk & 2.064 & 1.764 & 0.390 & -0.732 & 1.209 \\
\hline Agr & 1.703 & 2.598 & 0.837 & 2.649 & 0.991 \\
\hline Foo & 6.341 & 6.150 & 1.866 & 6.799 & 0.794 \\
\hline
\end{tabular}

Tabela 3. Variação das exportações totais (\%).

Em termos gerais, quando as exportações superam as importações é um indicativo de uma vantagem comparativa para a macrorregião com mais exportações. Essa vantagem comparativa foi ocasionada, somente, pelo choque no custo de frete, assim, o impacto dar-se-á no custo de produção menor para a região de maior vantagem comparativa, dado que o frete ou o transporte se torna componente no custo de produção para a agropecuária. Para Almeida e Guilhoto. (2007) e Branco et al. (2012), o custo de transporte impacta diretamente no custo produção agropecuária, por vezes, inviabilizando a produção local. O transporte passa a ser um fator limitador ao comércio entre as regiões por seu custo, conforme apontado por Inklaar e Timmer (2014). Ao elevar os custos de transporte de um ponto a outro, alguns produtos e serviços passam a ser inviáveis economicamente para uma região impedindo o comércio entre elas.

Assim, a região com menor custo de produção e, consequentemente, maior vantagem comparativa, terá um aumento na exportação dos produtos frente as regiões de menor vantagem comparativa. Tal fato é observado para todas a macrorregiões brasileiras.

Ao se correlacionar a vantagem comparativa e o desequilíbrio afetado setores o frete pode influenciar os preços finais, uma vez que a região com menores custos de produção terá melhor desempenho, ao contrário daqueles com maiores custos de produção.

Tal situação provoca uma perda de vantagem comparativa da qual a região com o menor custo de produção, incluindo o frete, deteria frente aquela de maior custo. Dessa forma, as preocupações apontadas por Fujita et al. (2000) e Oliveira et al. (2015), cuja perda de vantagem comparativa devido ao custo de transporte se torna reais, a medida em que o custo com o transporte gera ineficiência para um determinado setor, inviabilizando a produção ou - abastecimento de matéria prima e insumos produtivos na cadeia e tão logo é ocasionado uma perda de competitividade.

Considerando o frete como componente do custo de produção é normal que os setores tentem minimizar custos, em especial os setores que envolvem commodities agrícolas muito competitivas e de baixa margem de lucro. O aumento do custo de transporte, tanto para produtos finais quanto para insumos produtivos, pode afetar e até tornar os produtos inviáveis em determinadas regiões. Nas commodities, o frete e o preços devem ser analisados em conjunto, a fim de observar se o aumento do custo de transporte refletirá nos preços finais, de forma a se verificar se há transferência do aumento do custo de transporte para o consumidor.

\begin{tabular}{lccccc}
\hline & Norte & Nordeste & Centro-oeste & Sudeste & Sul \\
\hline Pdr & 0.172 & 0.039 & -0.174 & -0.084 & -0.006 \\
\hline Gro & 0.146 & 0.034 & -0.175 & -0.152 & -0.046 \\
\hline Osd & 0.200 & 0.063 & -0.194 & -0.043 & 0.019 \\
\hline c_b & 0.141 & 0.024 & -0.194 & -0.138 & -0.067 \\
\hline Oap & 0.175 & 0.060 & -0.186 & -0.069 & -0.019 \\
\hline rmk & 0.213 & 0.096 & -0.137 & -0.131 & 0.022 \\
\hline Agr & -0.437 & -0.195 & -0.330 & -0.276 & -0.268 \\
\hline Foo & 0.079 & -0.114 & -0.657 & -0.530 & -1.295 \\
\hline
\end{tabular}

Tabela 4. Variação de preços (\%). 
Ao se refere a preços, todas as alterações são inferiores a $1 \%$ causando uma diferença entre o comportamento nos preços do frete (com base nos preços dos combustíveis) e os preços finais dos bens. Isto pode indicar duas situações:(i) frete não é um custo importante e não afeta diretamente os preços finais ao consumidor; ou (ii) o Brasil não tem impacto para liderar as mudanças de preços no mercado de produtos agropecuários e é um tomador de preços internacionais.

Na primeira situação, existe alguns componentes de produção de custos e o frete é apenas mais um deles. No entanto, a segunda situação torna-se mais adequada, o que significa que a economia brasileira não é forte suficiente para mudar de preço do bem de maneira individual, principalmente se o setor está condicionado pelos preços internacionais das commodities, embora seja considerado um dos principais produtores e exportadores de commodities agrícolas do mundo.

Por se tratar de commodities agrícolas, há uma margem estreita de lucros e se atrela as commodities internacionais das quais muitas, como soja e milho, são negociadas em bolsa de mercadorias e futuros (com contrato a termo ou futuro), indicando uma trava de preços a médio e longo prazo de modo que seu preço final não seja impactado por somente uma variável de seu custo de produção.

A explicação para a variação das importações, exportações e preços são explicadas pela teoria microeconômica presente no modelo de EGC e se baseia no deslocamento da curva de oferta dos produtos agropecuários para a direita devido ao aumento da exportação e importação nas macrorregiões, conforme a Figura 7.

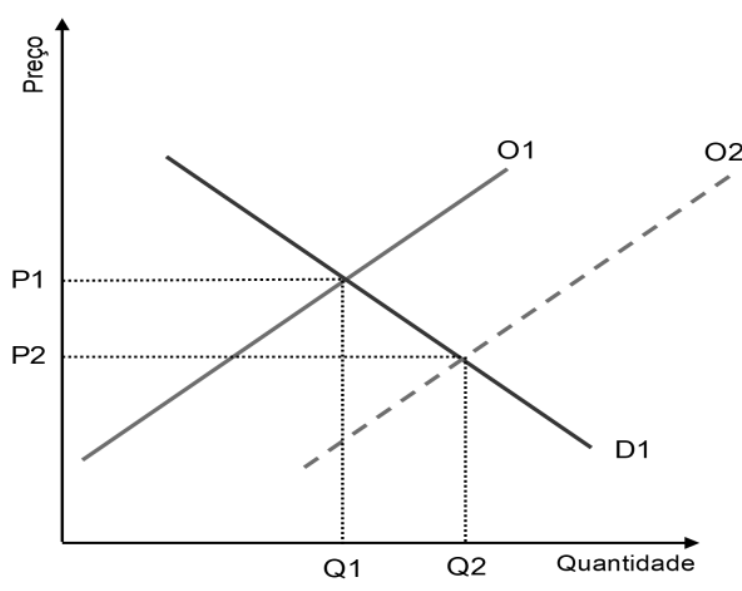

Figura 7. Deslocamento das curvas de oferta e demanda quando se aumenta as importações e exportações com a diminuição o preço.

No sul, sudeste e centro-oeste a diminuição dos preços dos produtos agrícolas foi provocado pelo deslocamento da curva de importação e exportação para a direita, aumentando a quantidade de produtos importados e exportados $(\mathrm{Q} 1<\mathrm{Q} 2)$ e a diminuição dos preços (P1 > P2). Isto representa um efeito direto da diminuição dos custos de transportes, uma vez que o custo logístico é componente relevante no preço final dos produtos segundo Castro (2009) e Caixeta Filho (2010).

Ao contrário do que ocorre quando há diminuição dos preços, o aumento é provocado pelo deslocamento da curva de oferta de produtos agropecuários para exportação e importação para a esquerda, diminuindo a quantidade ofertada e aumentando-se os preços. Esse movimento de aumento nos preços pode ser observado nas regiões norte e nordeste.

No caso de diminuição de preços, as macrorregiões brasileiras ganham em competitividade, já quando há aumento nos preços praticados, existe a perda na competitividade dos setores agropecuários. Tal fato é direcionando pela vantagem competitiva gerada na oscilação do custo de produção Assim, o menor custo de produção 
impactaria em uma maior vantagem comparativa na produção de produtos agropecuários.

Em suma, o comportamento do frete impacta diretamente nos fluxos comerciais inter-regionais brasileiros provocando deslocamento no comércio para aquelas regiões onde se tem maior vantagem comparativa.

\section{Considerações finais}

O objetivo do trabalho foi realizar um estudo sobre os impactos da oscilação do custo do frete para os produtos agropecuários brasileiros utilizando modelagem em Equilíbrio Geral Computável, tendo em vista o comportamento de preço, importação e exportação.

Através da modelagem em EGC o trabalho contribui com uma abordagem diferente de métodos mais tradicionais, criando uma alternativa para análise de como o custo de transporte impacta no comércio inter-regional.

Como resultado da projeção, a importação e a exportação sofreram impacto causado pela variação do preço do frete. Em casos, especialmente na mãode-obra intensiva, como a agricultura, as exportações e importações aumentam em todas as macrorregiões brasileiras para os produtos agropecuários. Esse tipo de variação é um indicativo no movimento dos fluxos comerciais e traz benefícios para a economia do país, uma vez que há atividade comercial dentro e fora do

\section{Referências}

Agência Nacional do Petróleo, Gás Natural E Biocombustíveis. (2019). Pesquisa mensal de preço de combustíveis. Brasília.

De Almeida, E. S., \& Guilhoto, J. J. M. (2007). O custo de transporte como barreira ao comércio na integração econômica: o caso do Nordeste. Revista Econômica do Nordeste, 38(2), 224-243..

ANTÓN, F. R. Logística del transporte. Universitat Politecnica de Catalunya. Iniciativa Digital Politecnica, 2005.

Asai, G., Bidarra, Z. S., \& Pinela, S. R. D. S. (2017). ANÁLISE DOS INVESTIMENTOS EM LOGÍSTICA DO PROGRAMA DE ACELERAÇÃO DO CRESCIMENTO (PAC): POLÍTICAS PÚBLICAS NA DIREÇÃO país, favorecendo a troca e o aquecimento da economia regional.

O deslocamento das curvas de oferta dos produtos agropecuários brasileiros indica um aumento nas importações e exportações pelas macrorregiões, impactando diretamente nos preços praticados. Quando se tem o deslocamento da curva de oferta para a direita, o preço tende a sofrer impacto negativo, ou seja, há sua diminuição, casos observados nas regiões sul, sudeste e centro-oeste. Nas regiões norte e nordeste, onde os preços aumentaram, houve o deslocamento da curva de oferta para a esquerda.

Este movimento da curva de oferta implica diretamente na competitividade do setor na microrregião. Quando existe o aumento da oferta impacta na diminuição dos preços e no aumento das exportações, indicativo de aumento da competitividade do setor que, mesmo com o aumento no valor do transporte (frete), aumenta sua competitividade ao gerenciar melhor os custos de produção frente as demais regiões.

Assim, o frete pode interferir nos fluxos comerciais inter-regionais. 0 cenário de comportamento do frete ao longo do tempo pode determinar um ponto de que a taxa de frete é importante para a economia nacional, impactando na competitividade dos setores agropecuários nas microrregiões do Brasil.

\section{CERTA?. Seminário Internacional sobre Desenvolvimento Regional. UNISC.}

Attavanich, W., McCarl, B. A., Ahmedov, Z., Fuller, S. W., \& Vedenov, D. V. (2013). Effects of climate change on US grain transport. Nature Climate Change, 3(7), 638-643.

Barry, M. P. (2013). US Global Logistics and Transport a Computable General Equilibrium Model.

Behar, A., \& Venables, A. J. (2011). Transport costs and international trade. Handbook of transport economics, 97-115.

Bosona, T., \& Gebresenbet, G. (2013). Food traceability as an integral part of logistics management in food and agricultural supply chain. Food control, 33(1), 32-48. 
Impactos no Comportamento do Frete: Uma Aplicação de Equilíbrio Geral Computável para os Produtos Agropecuários do Brasil

Bozoky, M. J., Oliveira, A. A. P., Deliberador, L. R., Formigoni, A., \& Jacubavicius, C. (2014). Análise do modal ferroviário no transporte de soja do centro oeste aos portos. INOVAE-Journal of Engineering, Architecture and Technology Innovation (ISSN $2357-$ 7797), 2(1), 50-61.

Branco, J. E. H., Caixeta Filho, J. V., Gameiro, A. H., Xavier, C. E. O., Pinheiro, M. A., \& de Souza, W. A. (2012). Otimização logística para o transporte multimodal de safras agrícolas no Brasil com foco no corredor Nordeste. Revista econômica do nordeste, 43(1), 67-92.

Bröcker, J. (2004). Computable general equilibrium analysis in transportation economics. Chapter, 16, 269-289. In Hensher, D. A; Button, K. J; Haynes, K. E; Stopher, P R. Handbook of Transport Geography and Spatial Systems (Handbooks in Transport, Volume 5).

Brooke, A., Kendrick, D., Meeraus, A. G. A. M. S., Raman, R., \& Rasenthal, R. E. (1998). : a User's Guide.'. GAMS Development Corporation: Washington, $D C$.

Caixeta Filho, J. V. (2010). Logística para a agricultura brasileira. Revista Brasileira de Comércio Exterior, 103, 18-30. .

Castro, N. (2009). Infra-estrutura de transporte e expansão da agropecuária brasileira. Planejamento e políticas públicas, (25). .

Christopher, M. (2016). Logistics \& supply chain management. Pearson UK.

Chen, Z., \& Rose, A. (2018). Economic resilience to transportation failure: a computable general equilibrium analysis. Transportation, 45(4), 10091027.

Cooper, M. C., Lambert, D. M., \& Pagh, J. D. (1997). Supply chain management: more than a new name for logistics. The international journal of logistics management, 8(1), 1-14.

CONFEDERAÇÃO

TRANSPORTE.(2014). Plano CNT de transporte e logística 2014. Brasília: CNT.
COUNCIL OF SUPPLY CHAIN MANAGEMENT PROFESSIONALS. (2013). Supply Chain Management Terms and Glossary. Illinois: CSCMP.

Crainic, T. G. (2003). Long-haul freight transportation. In Handbook of transportation science (pp. 451-516). Springer, Boston, MA.

Crainic, T. G., \& Laporte, G. (1997). Planning models for freight transportation. Design and Operation of Civil and Environmental Engineering Systems, 343.

Daskin, M. S. (1985). Logistics: an overview of the state of the art and perspectives on future research. Transportation Research Part A: General, 19(5-6), 383-398.

Fagundes, M. B. B., Dias, D. T., Pereira, M. W. G., Figueiredo Neto, L. F., \& Frainer, D. M. (2015). Impactos da produção de soja na economia de Mato Grosso do Sul. Revista de Política Agrícola, 23(4), 111122.

Fujita, M., \& Krugman, I. (2000). Economía espacial: las ciudades, las regiones y el comercio internacional (No. 330.9 F8Y.).

Gurgel, A. C. (2014). Impactos de políticas comerciais e agrícolas sobre a agropecuária e agroindústria brasileiras. In Anais... do 52ㅇ Congresso da Sociedade Brasileira de Economia e Sociologia Rural. Goiânia.

Gurgel, A. C., Pereira, M. W. G., \& Teixeira, E. C. (2013). A estrutura do PAEG. PAEG Technical Paper, (1).

Haddad, E. A. (2004). Retornos crescentes, custo de transporte e crescimento regional (Doctoral dissertation, Universidade de São Paulo). .

Inklaar, R., \& Timmer, M. P. (2014). The relative price of services. Review of Income and Wealth, 60(4), 727-746.

Kato, J. M. (2007). Um modelo para a construção de cenários aplicado à Indústria de Transportes Rodoviários de Cargas no Brasil. Revista da FAE, 10(2). . 
Kawano, B. R., Mores, G. D. V., Silva, R. F. D., \& Cugnasca, C. E. (2012). Estratégias para resolução dos principais desafios da logística de produtos agrícolas exportados pelo Brasil. Revista de Economia e Agronegócio/Brazilian Review of Economics and Agribusiness, 10(822-2016-54244), 71-88. .

Kim, E., Hewings, G. J., \& Amir, H. (2017). Economic evaluation of transportation projects: An application of Financial Computable General Equilibrium model. Research in Transportation Economics, 61, 44-55.

Lewczuk, K., \& Wasiak, M. (2011, August). Transportation services costs allocation for the delivery system. In 2011 21st International Conference on Systems Engineering (pp. 429-433). IEEE.

Lima, M. P. (2006). Custos logísticos na economia brasileira. Revista Tecnologística, 11(122), 64-69.

Machado, M. L. L., de Lima Oliveira, R., Ortiz, R., \& Colombelli, G. L. (2018). As principais dificuldades do modal de transporte rodoviário no Brasil. Revista da Mostra de Iniciação Científica e Extensão, 4(1).

Marchetti, D. D. S., \& Ferreira, T. T. (2012). Situação atual e perspectivas da infraestrutura de transportes e da logística no Brasil.

Marchetti, D. D. S., \& Ferreira, T. T. (2012). Situação atual e perspectivas da infraestrutura de transportes e da logística no Brasil. Brasília.

Nascimento, S. D., Gallon, A. V., \& Beuren, I. M. (2010). Formação de preços em empresa de transporte rodoviário de cargas.pensar contabil, 11(46)..

Péra'1, T. G., Guimarães, A. G., Lacerda, M. C. S. A., Gameiro', A. H., \& Caixeta-Filho', J. V. (2013). Modelo econométrico dos determinantes de preços de fretes rodoviários de cargas agrícolas envolvendo legislação, qualidade da via e multimodalidade. Associação de Pesquisa e Ensino em Transportes-ANPET..

Piercy, J. E., \& Ballou, R. H. (1978). A performance evaluation of freight transport modes. Logistics and Transportation Review, 14(2).
Oliveira, A. L. R., Cicolin, L., \& dos Santos, M. C. (2013). Estimativa do custo rodoviário da soja: uma análise da rota Sorriso-Santos. Revista de Economia e Agronegócio, 11(2).

Oliveira, J. S., de Matos, A. C., \& da Rocha, W. F. (2015). Estudo dos Fatores Determinantes do Preço do Frete Rodoviário no Escoamento da Soja a Granel da Mesorregião Oeste do Paraná com Destino ao Porto de Paranaguá nos Anos 2011, 2012 e 2013. Seminário Internacional sobre Desenvolvimento Regional.

Oliveira, A. L. R., Miyamoto, B., \& de Campos Coleti, J. ANÁLISE DOS DETERMINANTES DO VALOR DO FRETE DE GRÃOS NA REGIÃO CENTRO-SUL DO BRASIL. In: Congresso Nacional de Pesquisa em Transporte da ANPET, 31, Recife.

Sadoulet, E., \& De Janvry, A. (1995). Quantitative development policy analysis (Vol. 5). Baltimore: Johns Hopkins University Press.

Redding, S. J., \& Turner, M. A. (2015). Transportation costs and the spatial organization of economic activity. In Handbook of regional and urban economics (Vol. 5, pp. 1339-1398). Elsevier.

Rutherford, T. F. (2005). GTAP6inGAMS: The dataset and static model. Ann Arbor, MI.

Rutherford, T. F., \& Paltsev, S. V. (2000). GTAPinGAMS and GTAP-EG: global datasets for economic research and illustrative models. University of Colorado.

Rutherford, T. F. (1999). Applied general equilibrium modeling with MPSGE as a GAMS subsystem: An overview of the modeling framework and syntax. Computational economics, 14(1-2), 1-46.

Rushton, A., Croucher, P., \& Baker, P. (2014). The handbook of logistics and distribution management: Understanding the supply chain. Kogan Page Publishers..

Rutner, S. M., \& Langley, C. J. (2000). Logistics value: definition, process and measurement. The International Journal of Logistics Management.

Tavasszy, L. A., Ruijgrok, C., \& Thissen, M. J. P. M. (2003). Emerging global logistics networks: 
Impactos no Comportamento do Frete: Uma Aplicação de Equilíbrio Geral Computável para os Produtos Agropecuários do Brasil

implications for transport systems and Waquil, P. D. (2019). Alocação ótima de produtos policies. Growth and Change, 34(4), 456-472. agropecuários no Mercosul: um modelo de equilíbrio espacial com produtos intermediários. Revista de

Teixeira, E. C., Pereira, M. W., \& Gurgel, A. C. (2013). A estrutura do PAEG. Campo Grande: Life Editora.

Economia e Sociologia Rural, 34(1 e 2), 87-109.

Wilson, W. W., \& Dahl, B. (2011). Grain pricing and transportation: dynamics and changes in markets. Agribusiness, 27(4), 420-434.

\section{Reconhecimento}

Este estudo foi financiado em parte pela Coordenação de Aperfeiçoamento de Pessoal de Nível Superior Brasil (Capes) - Código financeiro 001.

\section{Sobre os autores}

Guilherme Asai - Universidade Estadual do Oeste do Paraná - UNIOESTE, Cascavel, PR (Brasil). Email: guilherme.asai@yahoo.com Orcid id: https://orcid.org/0000-0002-2880-8832

Carlos Alberto Piacenti - Universidade Estadual do Oeste do Paraná - UNIOESTE, Cascavel, PR (Brasil). Email: carlos.piacenti@unioeste.br Orcid id: https://orcid.org/0000-0001-6874-7379

Angelo Costa Gurgel - Fundação Getúlio Vargas - FGV, São Paulo, SP (Brasil). Email: angelo.gurgel@fgv.br Orcid id: https://orcid.org/0000-0001-8331-9508 


\title{
FREIGHT BEHAVIOR IMPACTS: AN APPLICATION OF COMPUTABLE GENERAL EQUILIBRIUM TO BRAZILIAN AGRICULTURAL PRODUCTS
}

\author{
Guilherme Asai, Carlos Alberto Piacenti, ${ }^{\Omega}$ Angelo Costa Gurgel \\ Universidade Estadual do Oeste do Paraná - UNIOESTE, Cascavel, PR (Brasil) \\ $\Omega$ Fundação Getúlio Vargas - FGV, São Paulo, SP (Brasil)
}

\section{ARTICLE DETAILS}

\section{Article history:}

Received: 08 July 2019

Accepted: 01 June 2020

Available online August: 01 th 2020

\section{Double Blind Review System}

\section{Scientific Editor}

Ilan Avrichir

\section{Key words}

Computable General Equilibrium

Logistics

Freight

Agriculture

\begin{abstract}
Objective: this study aimed to investigate the impact of the fluctuation in freight costs for Brazilian agricultural products.

Method: this is a descriptive research of a quantitative nature, focused on scenario analysis through modeling in computable general equilibrium for the five Brazilian macro-regions.

Main results: as a result of the research, it is indicated: (i) import and export suffered impact, caused by the fluctuation of the freight price, increasing in all Brazilian macro-regions; (ii) the increase in imports and exports is indicative that exist movement in trade flows and brings benefits to the country's economy, favoring the exchange and the regional economy and (iii) the regions with lower freight costs, gains of competitiveness for agricultural products.

Relevance/originality: this work contributes to a research agenda that involves important sectors of the Brazilian economy - agriculture and transport - and how they are related to each other, providing a view of how the freight cost influences in heating of the regional economy agricultural products trades in Brazil.

Theoretical/methodological contributions: the work presents a different approach than more traditional methods, by ECG, creating an alternative for analyzing how the cost of transport impacts on interregional trade.
\end{abstract}




\section{IMPACTOS EN EL COMPORTAMIENTO DEL CARGA: UNA APLICACIÓN DEL EQUILIBRIO GENERAL COMPUTABLE A LOS PRODUCTOS AGRÍCOLAS BRASILEÑOS}

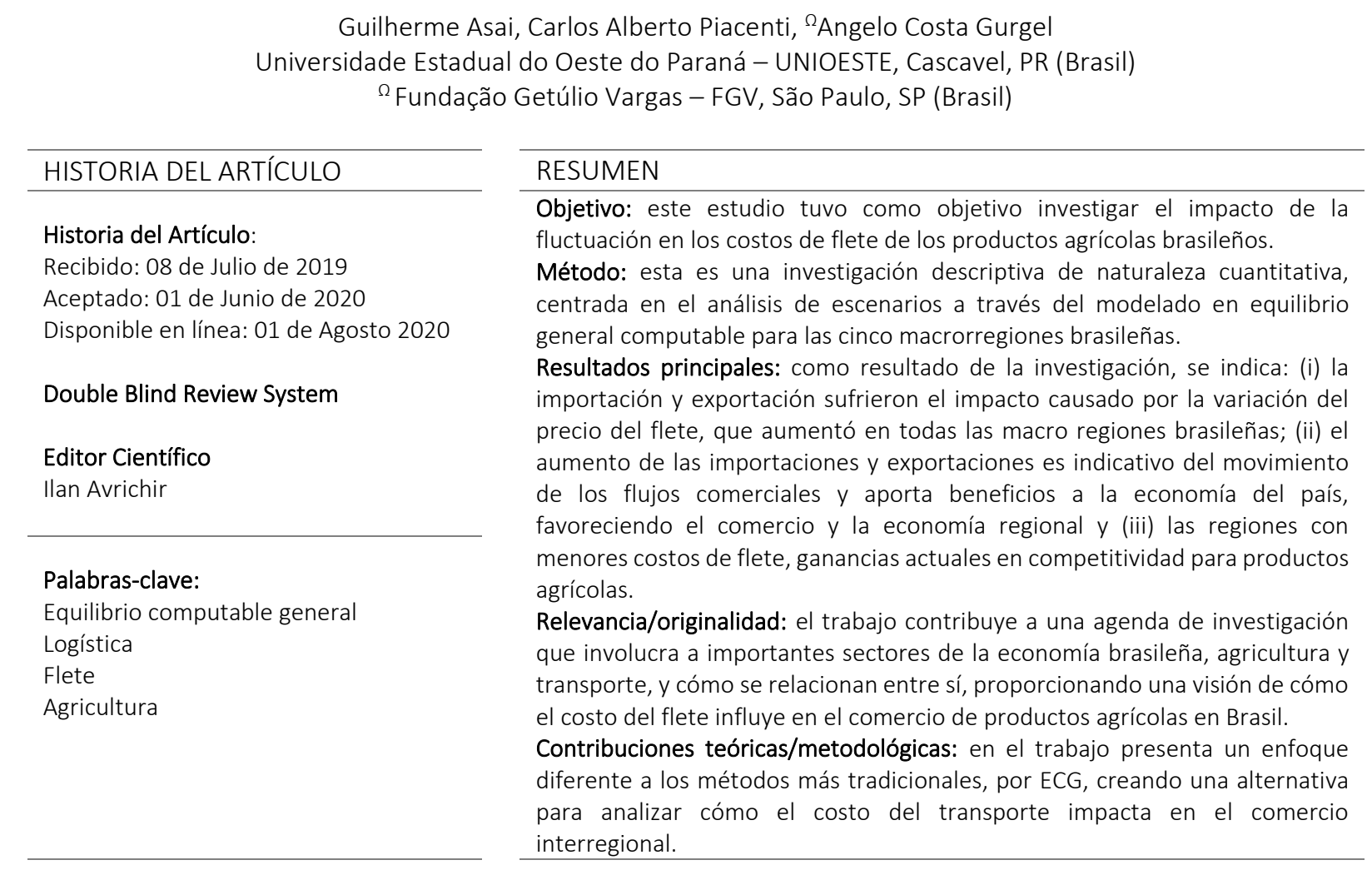

\section{Como citar este artigo:}

Asai, G., Piacenti, C., \& Gurgel, A. (2020). Impactos no Comportamento do Frete: Uma Aplicação de Equilíbrio Geral Computável para os Produtos Agropecuários do Brasil. Internext, 15(3), 17-33. doi:http://dx.doi.org/10.18568/internext.v15i3.556 